\title{
Complex Spectral Minutiae Representation For Fingerprint Recognition *
}

\author{
Haiyun Xu and Raymond N.J. Veldhuis \\ Department of Electrical Engineering, University of Twente \\ 7500 AE Enschede, The Netherlands \\ $\{\mathrm{h} . \mathrm{xu}, \mathrm{r.n} \cdot \mathrm{j} \cdot \mathrm{vel}$ dhuis\}@ewi.utwente.nl
}

\begin{abstract}
The spectral minutiae representation is designed for combining fingerprint recognition with template protection. This puts several constraints to the fingerprint recognition system: first, no relative alignment of two fingerprints is allowed due to the encrypted storage; second, a fixed-length feature vector is required as input of template protection schemes. The spectral minutiae representation represents a minutiae set as a fixed-length feature vector, which is invariant to translation, rotation and scaling. These characteristics enable the combination of fingerprint recognition systems with template protection schemes and allow for fast minutiae-based matching as well. In this paper, we introduce the complex spectral minutiae representation (SMC): a spectral representation of a minitiae set, as the locationbased and the orientation-based spectral minutiae representations (SML and SMO), but it encodes minutiae orientations differently. SMC improves the recognition accuracy, expressed in term of the Equal Error Rate, about 2-4 times compared with SML and SMO. In addition, the paper presents two feature reduction algorithms: the Column$P C A$ and the Line-DFT feature reductions, which achieve a template size reduction around $90 \%$ and results in a 1015 times higher matching speed (with 125,000 comparisons per second).
\end{abstract}

\section{Introduction}

Fingerprint recognition systems have the advantages of both ease of use and low cost. Nowadays, most fingerprint recognition systems are based on minutiae matching [9]. However, minutiae-based fingerprint matching algorithms have some drawbacks that limit their application.

First, due to the fact that minutiae sets are unordered, the correspondence between individual minutia in two minutiae sets is unknown before matching and this makes it

${ }^{*}$ This research is supported by the ProBiTe project funding by Sentinels and the TURBINE project funding by the European Union under the Seventh Framework Programme. difficult to find the geometric transformation that optimally registers (or aligns) two sets. This registration challenge causes minutiae-based matching algorithms to become rather slow. For fingerprint identification systems with very large databases [1], in which a fast comparison algorithm is necessary, minutiae-based matching algorithms will fail to meet the high performance speed requirement.

Second, the increasing widespread use of biometrics has raised substantial privacy concerns [6]. Researchers have shown the possibility of reconstructing fingerprints from minutiae templates [14]. Therefore, protecting minutiae templates becomes necessary. To combine fingerprint recognition with template protection, there are new constraints to the fingerprint recognition system: (1) no relative alignment of two fingerprints is allowed due to the encrypted storage; (2) the recently developed template protection schemes based on fuzzy commitment and helper data schemes, such as [15] and [7], require as an input a fixedlength feature vector representation of a biometric modality ${ }^{1}$.

There are several algorithms to extract a fixed-length feature vector from fingerprints. The FingerCode as presented in [5] is based on ridge features. The author concluded that FingerCodes are not as distinctive as minutiae and they can be used as complementary information for fingerprint matching. Willis and Myers brought forward a fixed-length minutiae wedge-ring feature [16], which recorded the minutiae numbers on a pattern of wedges and rings. However, this method can only perform a coarse fingerprint authentication, and cannot handle big translations and rotations. Park et al. proposed a feature vector based on the distribution of the pairwise distances between minutiae [12]. However, this algorithm is only evaluated on the manually labeled minutiae and the performance is not satisfying.

The spectral minutiae representation is a method that overcomes the drawbacks of the minutiae algorithms, thus broadening the application of minutiae-based algorithms [18]. This method represents a minutiae set as a

\footnotetext{
${ }^{1}$ Other template protection systems exist [10] that do not pose this fixed-length feature vector requirement.
} 
fixed-length feature vector, which is invariant to translation, and in which rotation and scaling become translations, so that they can be easily compensated for. These characteristics enable the combination of fingerprint recognition systems with template protection schemes and allow for faster matching as well. Moreover, the spectral minutiae representation method can be easily integrated into a minutiae-based fingerprint recognition system. Minutiae sets can be directly transformed to this new representation, which makes this method compatible with the large amount of existing minutiae databases.

In [18], the concept of the two representation methods are introduced: the location-based spectral minutiae representation (SML) that codes the minutiae locations, and the orientation-based spectral minutiae representation (SMO) that codes both minutiae locations and orientations. Although SMO incorporates the minutiae orientations, it did not show better results than SML in the experiments performed in [18]. This motivated us to design another spectral minutiae representation that incorporates minutiae orientations: the complex spectral minutiae representation (SMC). We denote it as complex in the sense that minutiae are represented as complex valued continuous functions in the spatial domain. We will also present two feature reduction algorithms designed for the spectral minutiae representations: the Column Principal Component Analysis (CPCA) and the Line Discrete Fourier Transform (LDFT) feature reduction algorithms. By applying feature reductions, we can reduce the template storage and at the same time increase the matching speed, which is a critical factor for many largescale biometric identification systems.

This paper is organized as follows. First, we give the background of the spectral minutiae representation and introduce the complex spectral minutiae representation in Section 2. Next, in Section 3, we present the Column-PCA and the Line-DFT feature reduction algorithms. Then, Section 4 shows the experimental results. Finally, we draw conclusions in Section 5.

\section{Spectral Minutiae Representations}

The objective of the spectral minutiae representation is to represent a minutiae set as a fixed-length feature vector, which is invariant to translation, rotation and scaling. In Figure 1, a general procedure of the spectral minutiae representation is illustrated. Step 1: we represent minutiae points as real (or complex) valued continuous functions, illustrated in Figure 1(b). In this representation, translation, rotation and scaling may exist, depending on the fingerprint sensors that have been used and how the user has put his finger on the sensor. Step 2: a two-dimensional continuous Fourier transform is performed and only the Fourier magnitude is kept, illustrated in Figure 1(c). This representation is now translation invariant according to the shift property of the

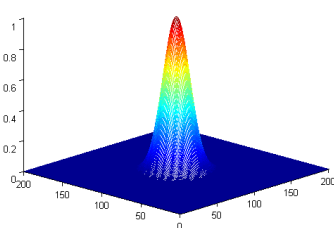

(a)

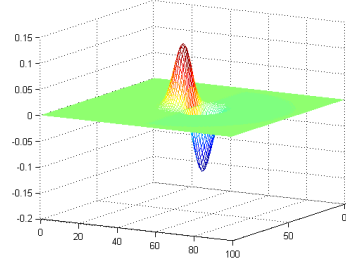

(b)
Figure 2. Representations of one minutiae point as a real valued continuous function. (a) SML; (b) SMO.

continuous Fourier transform. Step 3: the Fourier spectrum is re-mapped onto a polar-logarithmic coordinate system, illustrated in Figure 1(d). According to the scale and rotation properties of the two-dimensional continuous Fourier transform, now the rotation and scaling become translations along the new coordinate axes. It should be noted that this representation can be computed analytically. We will present the details later. In this paper, we will review SML and SMO, and then introduce SMC. These three representations are different in the "Step 1": SML and SMO represent minutiae points as real-valued continuous functions, while SMC represents minutiae as complex-valued continuous functions.

\subsection{Location-based spectral minutiae representa- tion (SML)}

Assume we have a fingerprint with $Z$ minutiae. In SML, we code the minutiae locations by indicator functions,

$$
m\left(x, y ; \sigma_{\mathrm{L}}^{2}\right)=\sum_{i=1}^{Z} \frac{1}{2 \pi \sigma_{\mathrm{L}}^{2}} \exp \left(-\frac{\left(x-x_{i}\right)^{2}+\left(y-y_{i}\right)^{2}}{2 \sigma_{\mathrm{L}}^{2}}\right),
$$

with $\left(x_{i}, y_{i}\right)$ the location of the $i$-th minutia in the fingerprint image. Thus, in the spatial domain, each minutia is represented by an isotropic two-dimensional Gaussian function, illustrated in Figure 2(a).

Taking the Fourier transform of $m\left(x, y ; \sigma_{\mathrm{L}}^{2}\right)$ and keeping only the magnitude of the Fourier spectrum (in order to make the spectrum invariant to translation of the input), we obtain the location-based spectral minutiae representation

$$
\begin{aligned}
& \mathcal{M}_{\mathrm{L}}\left(\omega_{\mathrm{x}}, \omega_{\mathrm{y}} ; \sigma_{\mathrm{L}}^{2}\right)= \\
& \left|\exp \left(-\frac{\omega_{\mathrm{x}}^{2}+\omega_{\mathrm{y}}^{2}}{2 \sigma_{\mathrm{L}}^{-2}}\right) \sum_{i=1}^{Z} \exp \left(-\mathrm{j}\left(\omega_{\mathrm{x}} x_{i}+\omega_{\mathrm{y}} y_{i}\right)\right)\right|
\end{aligned}
$$

\subsection{Orientation-based spectral minutiae represen- tation (SMO)}

The SML only uses the minutiae location information. However, including the minutiae orientation as well may 


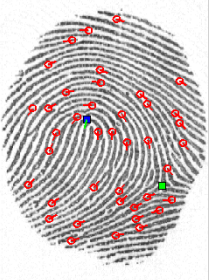

(a)

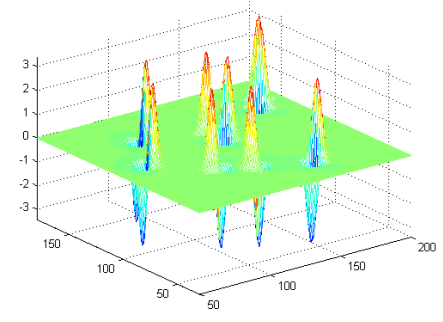

(b)

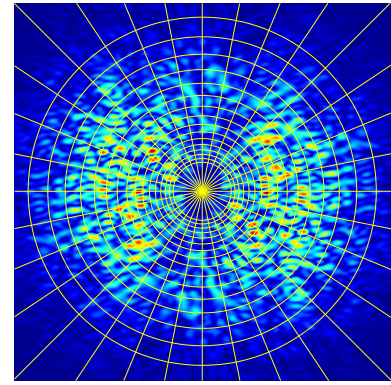

(c)

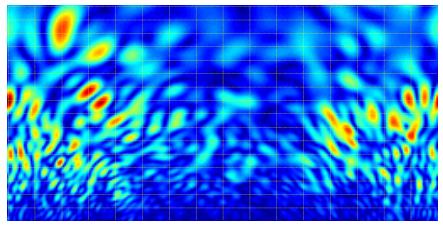

(d)

Figure 1. Illustration of the general spectral minutiae representation procedure (images from the SMO case). (a) a fingerprint and its minutiae; (b) representation of minutiae points as real (or complex) valued continuous functions; (c) the 2D Fourier spectrum of ' $b$ ' in a Cartesian coordinate and a polar-logarithmic sampling grid; (d) the Fourier spectrum sampled on a polar-logarithmic grid.

give better discrimination. Therefore, it can be beneficial to also include the orientation information in our spectral representation. In $\mathrm{SMO}$, the orientation $\theta$ of a minutia is incorporated by using the spatial derivative of $m(x, y)$ in the direction of the minutia orientation, illustrated in Figure 2(b). As with the SML algorithm, taking the magnitude of the Fourier spectrum yields

$$
\begin{aligned}
& \mathcal{M}_{\mathrm{O}}\left(\omega_{\mathrm{x}}, \omega_{\mathrm{y}} ; \sigma_{\mathrm{O}}^{2}\right)=\mid \exp \left(-\frac{\omega_{\mathrm{x}}^{2}+\omega_{\mathrm{y}}^{2}}{2 \sigma_{\mathrm{O}}^{-2}}\right) \\
& \times \sum_{i=1}^{Z} \mathrm{j}\left(\omega_{\mathrm{x}} \cos \theta_{i}+\omega_{\mathrm{y}} \sin \theta_{i}\right) \cdot \exp \left(-\mathrm{j}\left(\omega_{\mathrm{x}} x_{i}+\omega_{\mathrm{y}} y_{i}\right)\right) \mid .
\end{aligned}
$$

\subsection{Complex spectral minutiae representation (SMC)}

Although SMO incorporates the minutia orientation $\theta$, it did not show better results than SML in the experiments performed in [18]. The main reason is: in SMO, the minutiae orientation is incorporated as a derivative of the delta function, and this makes the minutiae noise (both in location and orientation) be amplified in the high frequency part of SMO. Therefore, a Gaussian kernel with higher $\sigma$ is needed for SMO to attenuate the noise in higher frequencies. However, the high frequency part also contains discriminative information, especially in case that the minutiae have good quality. This limitation of SMO motivated us to design another spectral minutiae representation that incorporates minutiae orientation: SMC.

In SMC, each minutia is first represented by an isotropic two-dimensional Gaussian function in the spatial domain (here it is the same as SML). Then we incorporate the minutiae orientation by assigning each Gaussian a complex amplitude $\mathrm{e}^{\mathrm{j} \theta_{i}}$, illustrated in Figure 3. This results in a phase shift in the frequency domain. Taking the magnitude of the Fourier spectrum yields

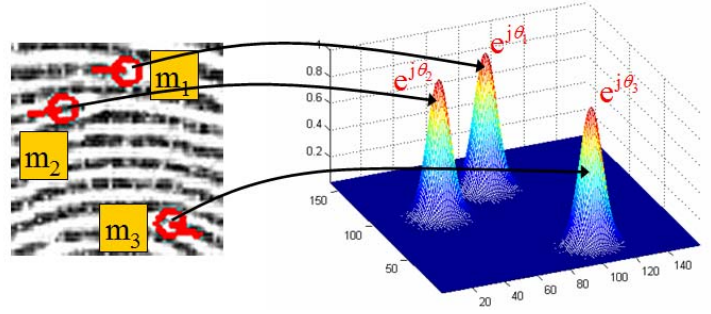

Figure 3. An illustration of three minutiae points represented as complex valued continuous functions.

$$
\begin{aligned}
& \mathcal{M}_{\mathrm{C}}\left(\omega_{\mathrm{x}}, \omega_{\mathrm{y}} ; \sigma_{\mathrm{C}}^{2}\right)= \\
& \left|\exp \left(-\frac{\omega_{\mathrm{x}}^{2}+\omega_{\mathrm{y}}^{2}}{2 \sigma_{\mathrm{C}}^{-2}}\right) \sum_{i=1}^{Z} \exp \left(-\mathrm{j}\left(\omega_{\mathrm{x}} x_{i}+\omega_{\mathrm{y}} y_{i}\right)+\mathrm{j} \theta_{i}\right)\right|
\end{aligned}
$$

\subsection{Polar-logarithmic (or polar) sampling}

In order to obtain the final spectral representations, the continuous spectra SML (2), SMO (3) and SMC (4) need to be sampled on a polar-logarithmic (or polar-linear) grid. A polar mapping transforms rotation to translation in the horizontal direction, while a logarithmic mapping transforms scaling to translation in the vertical direction ${ }^{2}$. In the radial direction $\lambda$, we use $M=128$ samples between $\lambda_{\mathrm{l}}$ and $\lambda_{\text {h. }}$. In the angular direction $\beta$, we use $N=256$ samples uniformly distributed between $\beta=0$ and $\beta=\pi$ or $2 \pi$ (because of the symmetry of the Fourier transform for realvalued functions, using the interval between 0 and $\pi$ for SML and SMO is sufficient). A polar-logarithmic sampling process is illustrated in Figures 1(c) and 1(d). The sampled

\footnotetext{
${ }^{2}$ In most fingerprint databases, there is no scaling difference between the fingerprints, or the scaling can be compensated for on the level of the minutiae sets [3]. Therefore, we sample SML and SMO in a polarlogarithmic grid in order to be consistent with [18], while we sample SMC in a polar-linear grid, which can provide more samples in the higher frequency part.
} 


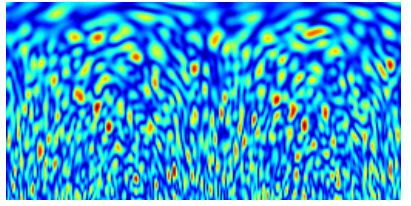

(a)

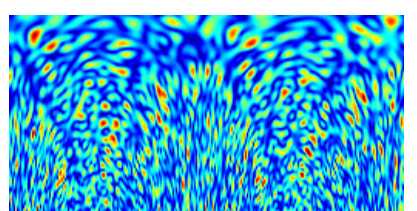

(c)

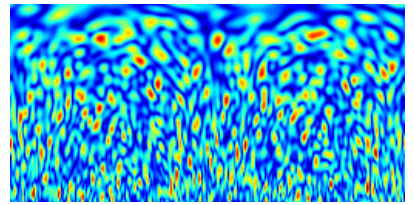

(b)

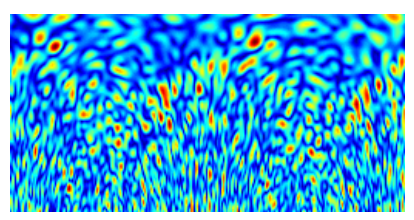

(d)
Figure 4. Examples of minutiae spectra using SMC. (a) and (b) are the SMC spectra from the same finger; (c) and (d) are the SMC spectra from the same finger.

spectra (2), (3) and (4) will be denoted by $S_{\mathrm{L}}\left(m, n ; \sigma_{\mathrm{L}}\right)$, $S_{\mathrm{O}}\left(m, n ; \sigma_{\mathrm{O}}\right)$ and $S_{\mathrm{C}}\left(m, n ; \sigma_{\mathrm{C}}\right)$, respectively. When no confusion can arise, the parameter $\sigma$ and the subscripts L, $\mathrm{O}$ and $\mathrm{C}$ will be omitted.

Examples of the minutiae spectra achieved with SMC are shown in Figure 4. For each spectrum, the horizontal axis represents the rotation angle of the spectral magnitude (from 0 to $2 \pi$ ); the vertical axis represents the frequency of the spectral magnitude (the frequency increases from top to bottom). It should be noted that the minutiae spectrum is periodic on the horizontal axis.

\subsection{Spectral Minutiae Matching}

Let $R(m, n)$ and $T(m, n)$ be the two sampled minutiae spectra, respectively, achieved from the reference fingerprint and test fingerprint. Both $R(m, n)$ and $T(m, n)$ are normalized to have zero mean and unit energy. We use the two-dimensional correlation coefficient between $R$ and $T$ as a measure of their similarity.

In practice, the input fingerprint images are rotated and might be scaled (for example, depending on the sensor that is used to acquire an image). Assume that the scaling has already been compensated for on the level of the minutiae sets [3]. Then we only need to test a few rotations, which become the circular shifts in the horizontal direction. We denote $T(m, n-j)$ as a circularly shifted version of $T(m, n)$. We use the fast rotation shift searching algorithm, based on variable stepsizes that was presented in $[20]^{3}$ and finally the maximum score from different combinations is the final matching score between $R$ and $T$,

\footnotetext{
${ }^{3}$ In [20], totaly 9 rotations are tested in a range of $-20^{\circ}$ to $+20^{\circ}$ in case of $N=256$ samples between 0 to $2 \pi$.
}

$$
\begin{array}{r}
S^{(R, T)}=\max _{j}\left\{\frac{1}{M N} \sum_{m, n} R(m, n) T(m, n-j)\right\}, \\
-15 \leq j \leq 15 .
\end{array}
$$

\section{Spectral Minutiae Feature Reduction}

The spectral minutiae feature is a 32,768 -dimensional real-valued feature vector. The large dimensionality of the spectral minutiae feature can cause three problems. First, the template storage requirement is very high. Second, the high dimensionality leads to a computational burden and the matching speed will be limited. Third, the high dimensionality can lead to a small sample size problem [13]. In order to cope with these problems, we introduce two feature reduction methods: the Column Principal Component Analysis (CPCA) and the Line Discrete Fourier Transform (LDFT) feature reduction algorithms, which can be applied in conjunction.

\subsection{Column-PCA feature reduction (CPCA)}

Principal component analysis (PCA) if often used in dimensionality reduction. However, there are two problems in implement PCA on the spectral minutiae representations. The first is the small sample size problem. An unreduced spectral minutiae representation has a dimensionality of $D=32,768$. A reliable PCA feature reduction requires a large number of fingerprint samples to implement the PCA training, which is difficult to acquire. The second problem is that the minutiae spectra are not rotationinvariant. As we mentioned in the previous section, the rotation of fingerprints becomes a circular shift in the horizontal direction. For the PCA training, all the minutiae spectra must be aligned beforehand in order to get meaningful results. Then both the training and matching processes become complicated. To cope with these problems, we introduce the Column-PCA method to perform a feature reduction.

We first look at the spectral minutiae feature $S$ in the 2D case as we presented in Section 2.4. From Figure 4, we can see that the minutiae spectrum is periodic on the horizontal axis. Moreover, on the vertical axis, the spectra with different frequencies are correlated. Therefore, we consider to use PCA to decorrelate the spectra with different frequencies in the vertical direction. To achieve this, we regard each column of $S$ as a new feature vector, thus $S=\left(\vec{z}_{1}, \ldots, \vec{z}_{N}\right)$, with $\vec{z}$ column feature vectors.

If we have $L$ samples $S_{1}, \ldots, S_{L}$ in the training set, we can create a $M \times L_{\mathrm{N}}\left(L_{\mathrm{N}}=N \times L, N=256\right)$ data matrix $\mathbf{Z}$ consists of all the samples, as $\mathbf{Z}=\left[\vec{z}_{1}, \ldots, \vec{z}_{L_{\mathrm{N}}}\right]$. To implement CPCA, we first subtract the sample mean (column mean) from the data matrix $\mathbf{Z}$. The next step is to apply 
SVD on $\mathbf{Z}$,

$$
\mathbf{Z}=\mathbf{U}_{\mathrm{Z}} \mathbf{S}_{\mathrm{Z}} \mathbf{V}_{\mathrm{Z}}^{\mathrm{T}}
$$

Finally we can obtain the CPCA transform matrix $\widetilde{\mathbf{U}}_{\mathrm{Z}}$ by retaining the first $M_{\mathrm{CPCA}}\left(M_{\mathrm{CPCA}} \leq M\right)$ columns of $\mathrm{U}_{\mathrm{Z}}$. The CPCA transform on the minutiae spectra $S(m, n)$ is written as

$$
\mathbf{S}_{\mathrm{CPCA}}=\widetilde{\mathbf{U}}_{\mathrm{Z}}^{\mathrm{T}} \mathbf{S}
$$

with $\mathbf{S}_{\mathrm{CPCA}}$ the $M_{\mathrm{CPCA}} \times N$ data matrix with reduced dimensions. After the CPCA feature reduction, the relation of the energy retainment rate $E_{\mathrm{CPCA}}$ and $M_{\mathrm{CPCA}}$ is

$$
E_{\mathrm{CPCA}}\left(M_{\mathrm{CPCA}}\right)=\frac{\sum_{n=1}^{M_{\mathrm{CPCA}}} \mathbf{S}_{\mathrm{Z}}(n, n)}{\sum_{n=1}^{M} \mathbf{S}_{\mathrm{Z}}(n, n)}, 1 \leq M_{\mathrm{CPCA}} \leq M,
$$

and the retained dimensionality at a target energy retainment rate $\tilde{E}_{\mathrm{CPCA}}$

$$
M_{\mathrm{CPCA}}\left(\tilde{E}_{\mathrm{CPCA}}\right)=\arg \min _{1 \leq \widetilde{M} \leq M}\left|\frac{\sum_{n=1}^{\widetilde{M}} \mathbf{S}_{\mathrm{Z}}(n, n)}{\sum_{n=1}^{M} \mathbf{S}_{\mathrm{Z}}(n, n)}-\tilde{E}_{\mathrm{CPCA}}\right| .
$$

The CPCA transform is illustrated in Figures 5(a) and 5(b). We can see that after the CPCA transform, the main energy of the original minutiae spectrum $S$ is concentrated in the top lines of $S_{\mathrm{CPCA}}$. By only retaining the top $M_{\mathrm{CPCA}}$ lines, we perform the CPCA feature reduction, with a reduction rate $R_{\mathrm{CPCA}}=\left(M-M_{\mathrm{CPCA}}\right) / M$. Because the rotation operator commutes with column transformation, the minutiae spectrum $S_{\mathrm{CPCA}}$ remains periodic on the horizontal axis after the CPCA transform.

\subsection{Line-DFT feature reduction (LDFT)}

The CPCA feature reduction method reduces the minutiae spectrum feature $S$ in the vertical direction. In this section, we will introduce the Line-DFT feature reduction (LDFT) method, which will reduce the feature in the horizontal direction. This method is based on the fact that the minutiae spectrum $S$ is periodic on the horizontal axis. Therefore, LDFT can be applied both independently and in combination with the CPCA.

We denote each line of the minutiae spectrum $S$ as a line feature vector $\vec{y}$, thus $S=\left(\vec{y}_{1}, \ldots, \vec{y}_{M}\right)^{\mathrm{T}}$. Because each line $y_{m}[n],(m=1, \ldots, M)$ is a periodic discrete-time signal, by

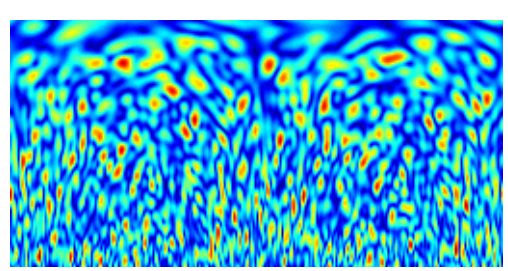

(a)

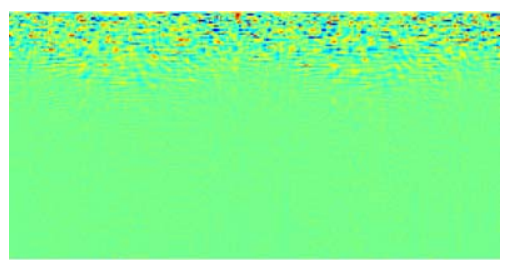

(b)

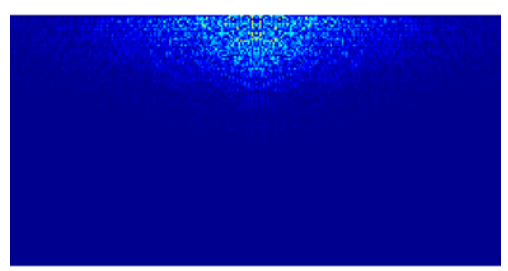

(c)

Figure 5. Illustration of the CPCA transform and the LDFT representation. (a) the complex spectral minutiae; (b) the minutiae spectrum after the CPCA transform; (c) the magnitude of the LDFT representation of (b).

performing DFT (implemented as a FFT) on each $y_{m}[n]$, we can obtain $S_{\mathrm{LDFT}}=\left(Y_{1}[k], \ldots, Y_{M}[k]\right)^{\mathrm{T}}, S_{\mathrm{LDFT}} \in \mathbb{C}^{M}$, which is an exact representation of $S$.

The LDFT representation is illustrated in Figures 5(b) and 5(c). We can see that after the LDFT representation, the main energy is concentrated in the low frequency part (the middle columns). Therefore, for each line of the LDFT representation $S_{\mathrm{LDFT}}$, we only retain the Fourier components with a certain percentage of energy (for example, $80 \%$ ) in the lower frequency part. By reducing the number of Fourier components, we implement the LDFT feature reduction. For each line $m$, the relation of the energy retainment rate $E_{\mathrm{LDFT}}$ after the LDFT feature reduction and $N_{\text {LDFT }}$ (which indicates that only the $N_{\text {LDFT }}$ Fourier components from the low frequency part are retained) is

$$
\begin{gathered}
E_{\mathrm{LDFT}}\left(N_{\mathrm{LDFT}} ; m\right)=\frac{\sum_{k=0}^{N_{\mathrm{LDFT}}-1}\left|Y_{m}(k)\right|^{2}}{\sum_{k=0}^{N / 2}\left|Y_{m}(k)\right|^{2}}, \\
1 \leq N_{\mathrm{LDFT}} \leq \frac{N}{2}+1,
\end{gathered}
$$

and the retained dimensionality at a target energy retain- 
ment rate $\tilde{E}_{\mathrm{LDFT}}$

$$
\begin{aligned}
& N_{\mathrm{LDFT}}\left(\tilde{E}_{\mathrm{LDFT}} ; m\right)= \\
& \arg \min _{1 \leq \tilde{N} \leq \frac{N}{2}+1}\left|\frac{\sum_{k=0}^{\tilde{N}-1}\left|Y_{m}(k)\right|^{2}}{\sum_{k=0}^{N / 2}\left|Y_{m}(k)\right|^{2}}-\tilde{E}_{\mathrm{LDFT}}\right| .
\end{aligned}
$$

As we mentioned in Section 2.5, the rotation of the fingerprint becomes the circular shift of the minutiae spectrum along the horizontal axis in the space domain. To test different fingerprint rotations (see Section 2.5) after applying the LDFT representation, we will implement the shift operation in the frequency domain according to the shift property of the discrete Fourier transform. Thus, the Line-DFT transformation of $T\left(m, n-n_{c s}\right)$ in Equation (5) becomes

$$
\begin{aligned}
& T\left(m, n-n_{c s}\right)=\left(y_{1}\left(n-n_{c s}\right), \ldots, y_{M}\left(n-n_{c s}\right)\right)^{\mathrm{T}} \\
& \stackrel{\mathrm{LDFT}}{\longrightarrow} \exp \left(-\mathrm{j} \frac{2 \pi}{N} k n_{c s}\right)\left(Y_{1}(k), \ldots, Y_{M}(k)\right)^{\mathrm{T}}
\end{aligned}
$$

The DFT is orthnormal, thus it preserves inner products. Consider two discrete-time, periodic signals $f_{1}[n]$ and $f_{2}[n], f_{1}[n], f_{2}[n] \in \mathbb{R}^{N}$, with period $N$ ( $N$ is an even number), because of the symmetry properties of the DFT for real-valued signals, the correlation of $f_{1}[n]$ and $f_{2}[n]$ becomes

$$
\begin{aligned}
& \sum_{n=0}^{N-1} f_{1}[n] f_{2}^{*}[n]=\frac{1}{N}\left(A_{1}[0] A_{2}[0]\right. \\
& \left.+2 \sum_{k=1}^{\frac{N}{2}-1}\left(A_{1}[k] A_{2}[k]+B_{1}[k] B_{2}[k]\right)+A_{1}\left[\frac{N}{2}\right] A_{2}\left[\frac{N}{2}\right]\right) .
\end{aligned}
$$

where $*$ denotes the complex conjugate, $\Re$ denotes the real part, $A_{i}[k]$ and $B_{i}[k]$ are the real and the imaginary part of the Fourier coefficients.

Equation (13) shows that we can generate two one dimensional real-valued feature vectors $\overrightarrow{v_{1}}$ and $\overrightarrow{v_{2}}$ from the Fourier components, that are,

$$
\begin{array}{r}
\overrightarrow{v_{i}}=\frac{1}{\sqrt{N}}\left(A_{i}[0], \sqrt{2} A_{i}[1], \ldots, \sqrt{2} A_{i}\left[\frac{N}{2}-1\right], A_{i}\left[\frac{N}{2}\right]\right. \\
\left.\sqrt{2} B_{i}[1], \ldots, \sqrt{2} B_{i}\left[\frac{N}{2}-1\right]\right), i=1,2 .
\end{array}
$$

The correlation of $\overrightarrow{v_{1}}$ and $\overrightarrow{v_{2}}$ is exactly the same as the correlation of the real-valued signals $f_{1}[n]$ and $f_{2}[n]$. Thus, we can continue using the correlation-based spectral minutiae matching algorithm. In the LDFT feature reduction, only the $N_{\text {LDFT }}\left(N_{\text {LDFT }} \leq \frac{N}{2}+1\right)$ Fourier components from the low frequency part are retained. For the matching algorithm presented in Section 2.5, we denote $v_{r}$ and $v_{t, n_{c s}}$ as the reduced features of $R(m, n)$ and $T\left(m, n-n_{c s}\right)$ respectively, then Equation (5) becomes

$$
S^{(R, T)}=\max _{n_{c s}}\left\{\frac{1}{M N} \sum v_{r} v_{t, n_{c s}}\right\},-15 \leq n_{c s} \leq 15 .
$$

\section{Experiments}

\subsection{Experimental settings}

The proposed algorithms have been evaluated on MCYT [11] and FVC2002-DB2 [8] fingerprint databases. The fingerprint data that we used from MCYT are obtained from 145 individuals (person ID from 0000 to 0144 and finger ID 0) and each individual contributes 12 samples. We use samples from person ID 0100 to 0144 for the CPCA and LDFT training (totally 540 fingerprints) and samples from person ID 0000 to 0099 for test (totally 1200 fingerprints). We also tested our algorithms on the FVC2002-DB2 because it is a public-domain fingerprint database. Compared with MCYT, the fingerprints in FVC2002 have lower quality and bigger displacements. For the FVC databases, we apply the same experimental protocol as in the FVC competition: the samples from finger ID 101 to 110 for the CPCA and LDFT training (totally 40 fingerprints) and samples from person ID 1 to 100 for test (totally 400 fingerprints $)^{4}$. The minutiae sets were obtained by the VeriFinger minutiae extractor $[2]^{5}$.

We test our algorithm in a verification setting. For matching genuine pairs, we used all the possible combinations. For matching imposter pairs, we chose the first sample from each identity. For the parameters $\sigma_{\mathrm{L}}, \sigma_{\mathrm{O}}$ and $\sigma_{\mathrm{C}}$ in Equations (2), (3) and (4), we chose $\sigma=0$ for SML and SMC (in this case, no multiplication with Gaussian in the frequency domain) and $\sigma=4.24$ for SMO (the explanation of parameter settings can be found in [18]). In our experiment, we also use the core as a reference point to assist the verification, following the procedure in $[18]^{6}$.

\footnotetext{
${ }^{4}$ We propose to use our algorithm in a high security scenario. In FVC2002 databases, samples 3, 4, 5 and 6 were obtained by requesting the users to provide fingerprints with exaggerated displacement and rotation. In a high security scenario where the user is aware that cooperation is crucial for security reasons, he will be cooperative. Therefore, only samples 1, 2, 7 and 8 are chosen. To deal with the large rotations, an absolute pre-alignment based on core and its direction can be applied.

${ }^{5}$ VeriFinger Extractor Version 5.0.2.0 is used.

${ }^{6}$ In [18], for each fingerprint, maximum two cores or/and two deltas were used to improve the performance. In this paper, only the upper core is used.
} 
Table 1. Parameters of CPCA and LDFT (MCYT database).

\begin{tabular}{|l|c|c|c|}
\hline Methods & SML & SMO & SMC \\
\hline \hline$E_{\text {CPCA }}$ & $83 \%$ & $90 \%$ & $84.7 \%$ \\
\hline Reduction & $78.1 \%$ & $78.1 \%$ & $75 \%$ \\
\hline \hline$E_{\text {LDFT }}$ & $99 \%$ & $99 \%$ & $75 \%$ \\
\hline Reduction & $67.8 \%$ & $72.8 \%$ & $58.3 \%$ \\
\hline \hline$E_{\text {Total }}$ & $82.2 \%$ & $63.5 \%$ & $85.6 \%$ \\
\hline Reduction & $92.9 \%$ & $94.0 \%$ & $89.6 \%$ \\
\hline
\end{tabular}

Table 2. Parameters of CPCA and LDFT (FVC2002-DB2 database)

\begin{tabular}{|l|c|c|c|}
\hline Methods & SML & SMO & SMC \\
\hline \hline$E_{\text {CPCA }}$ & $75 \%$ & $92 \%$ & $66.2 \%$ \\
\hline Reduction & $75 \%$ & $75 \%$ & $75 \%$ \\
\hline \hline$E_{\text {LDFT }}$ & $97 \%$ & $98 \%$ & $70 \%$ \\
\hline Reduction & $68.1 \%$ & $72.5 \%$ & $51.1 \%$ \\
\hline \hline$E_{\text {Total }}$ & $72.8 \%$ & $90.2 \%$ & $46.3 \%$ \\
\hline Reduction & $92.0 \%$ & $93.1 \%$ & $81.5 \%$ \\
\hline
\end{tabular}

Table 3. Results after CPCA and LDFT (MCYT database).

\begin{tabular}{|c|c|c|}
\hline Methods & EER & GAR @ FAR=0.1\% \\
\hline \hline SML & $0.67 \%$ & $98.8 \%$ \\
\hline SMO & $0.71 \%$ & $98.6 \%$ \\
\hline SMC & $0.16 \%$ & $99.8 \%$ \\
\hline Fusion SML and SMC & $0.06 \%$ & $99.9 \%$ \\
\hline
\end{tabular}

Table 4. Results after CPCA and LDFT (FVC2002-DB2 database).

\begin{tabular}{|c|c|c|}
\hline Methods & EER & GAR @ FAR $=0.1 \%$ \\
\hline \hline SML & $5.1 \%$ & $88.7 \%$ \\
\hline SMO & $4.51 \%$ & $86.6 \%$ \\
\hline SMC & $3.05 \%$ & $94.1 \%$ \\
\hline Fusion SML and SMC & $2.48 \%$ & $95.6 \%$ \\
\hline
\end{tabular}

\subsection{Results}

We test the SML, SMO and SMC representations in the two databases. We present the results in both with and without CPCA and LDFT cases to evaluate the performances of the feature reduction algorithms. During feature reduction, the selection of the energy retainment rates $E_{\mathrm{CPCA}}$ and $E_{\mathrm{LDFT}}$ are important for the performance. When $E_{\mathrm{CPCA}}$ and $E_{\mathrm{LDFT}}$ are chosen, we can calculate $M_{\mathrm{CPCA}}$ and $N_{\mathrm{LDFT} m}$ using the fingerprints in the training sets, according to Equations (9) and (11).

The feature reduction parameters are shown in Tables 1 and 2. We can see that regarding LDFT, SMC has lower reduction rates and energy retainment compared with SML and SMO. The reason is that SMC samples a $2 \pi$ range in the horizontal direction, while SML and SMO a range of $\pi$. Therefore, the horizontal feature reduction rates for SMC are lower.

The performances of SML, SMO, SMC and the feature

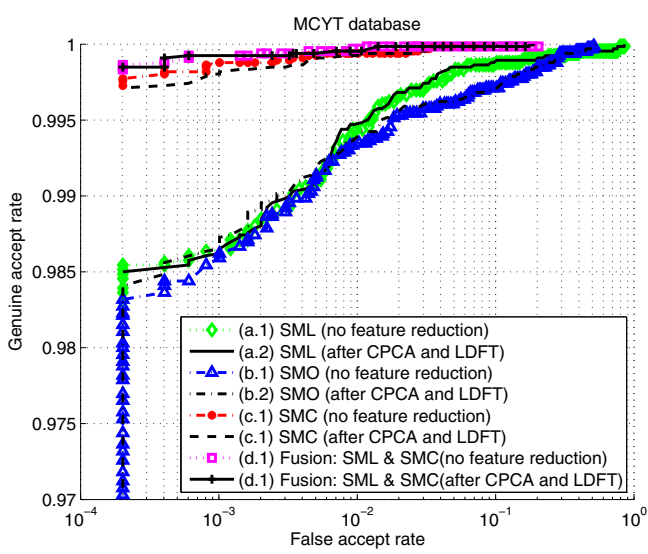

Figure 6. ROC curves (MCYT database).

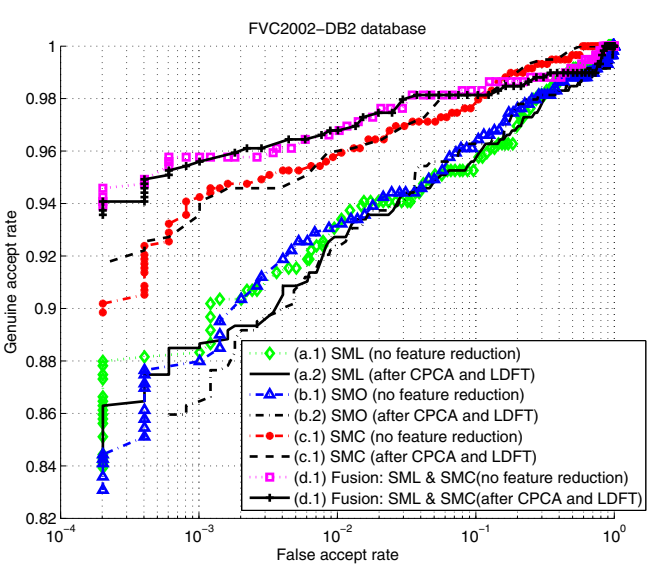

Figure 7. ROC curves (FVC2002-DB2 database).

reduction results are shown in Tables 3 and 4 and the ROC curves are in Figures 6 and 7. From the ROC curves, we can see that there is no noticeable performance degradation after the CPCA and LDFT feature reductions. By using both methods, we can achieve a template size reduction around $90 \%$.

From Tables 3 and 4, we can see that the recognition rates of SMC have substantial improvements compared with SML and SMO (the improvement factors range from 1.5 to 4.4 in the EERs). It is understandable that SMC outperformed SML because SMC incorporates the minutiae orientation information. As for SMO, we explained perviously that in the SMO representation, the critical information of minutiae orientations is in the high frequency region, where also contains more noise. While with SMC, this critical information is spread over the entire spectrum. This explains how the SMC overcomes the drawback of the SMO technique. A preliminary attempt of SML and SMC fusion (considering the recognition performances of SML and SMC, a score level sum-rule fusion with weights 1:2 
has been applied) is also applied and results in some clear improvements in accuracy.

Without feature reductions, we can implement 8,000 comparisons per second using optimized C language programming on a PC with Intel Pentium D processor 2.80 $\mathrm{GHz}$ and $1 \mathrm{~GB}$ of RAM. After applying CPCA and LDFT, we can implement 125,000 comparisons (the speed is more than 15 times faster) under the same setting. We also tested the VeriFinger matcher, a fast commercial minutiae-based matcher, using the same PC setting and the matching speed is 8,000 comparisons per second. Our matching speed will be slowed down by incorporating core information (reduces 2 times) and fusion of SML and SMC (reduces 2 times). After including these factors, our spectral minutiae matching still has speed advantages compared with most existing minutiae-based algorithms.

\section{Conclusions and future work}

Minutiae-based matching is the most widely used technique in fingerprint recognition systems. However, the low matching speed is limiting their application. At the same time, the increasing security and privacy concerns make minutiae template protection one of the most crucial tasks. The spectral minutiae representation has coped with the above issues.

In this paper, we present the complex spectral minutiae representation and the CPCA and LDFT feature reduction algorithms. These new techniques enhance the recognition accuracy and increase the matching speed as well, thus broaden the application of the spectral minutiae representation algorithm. In addition, our other preliminary research showed that we can further improve accuracy about $20 \%$ to $70 \%$ by applying minutiae quality data and minutiae subsets [17], [19]. We will continue exploring the potential of increasing recognition accuracy.

Furthermore, in order to be able to apply the spectral minutiae representation with a template protection scheme, for example based on a fuzzy extractor [4], the next step would be to extract bits that are stable for the genuine user and completely random for an arbitrary user. A fixed-length binary representation also has other advantages such as the small template storage and high matching speed. This will also be our future work.

\section{References}

[1] United States Visitor and Immigrant Status Indicator Technology Program (US-VISIT).

[2] VeriFinger SDK. http://www. neurotechnologija.com/.

[3] ISO/IEC 19794-2, Information Technology - Biometric Data Interchange Format - Part 2: Finger Minutiae Data. 2005.

[4] Y. Dodis, L. Reyzin, and A. Smith. Fuzzy extractors. In P. Tuyls, B. Skoric, and T. Kevenaar, editors, Security with
Noisy Data - On Private Biometrics, Secure Key Storage and Anti-Counterfeiting, chapter 3, pages 45-56. Springer, 2007.

[5] A. Jain, S. Prabhakar, L. Hong, and S. Pankanti. Filterbankbased fingerprint matching. IEEE Trans. Image Processing, 9(5):846-859, May 2000.

[6] A. K. Jain, K. Nandakumar, and A. Nagar. Biometric template security. EURASIP J. Adv. Signal Process, 2008:1-17, 2008.

[7] A. Juels. Fuzzy commitment. In P. Tuyls, B. Skoric, and T. Kevenaar, editors, Security with Noisy Data - On Private Biometrics, Secure Key Storage and Anti-Counterfeiting, chapter 3, pages 45-56. Springer, 2007.

[8] D. Maio, D. Maltoni, R. Cappelli, J. Wayman, and A. Jain. FVC2002: Second fingerprint verification competition. 3:811-814, Aug. 2002.

[9] D. Maltoni, D. Maio, A. Jain, and S. Prabhakar. Handbook of Fingerprint Recognition. Springer, New York, 2003.

[10] K. Nandakumar, A. Jain, and S. Pankanti. Fingerprint-based fuzzy vault: Implementation and performance. Information Forensics and Security, IEEE Transactions on, 2(4):744757, Dec. 2007.

[11] Ortega-Garcła, J., et al. MCYT baseline corpus: a bimodal biometric database. In IEE Proc. Vision, Image and Signal Processing 150(6), pages 395-401, 2003.

[12] C.-H. Park, M. J. T. Smith, M. Boutin, and J.-J. Lee. Fingerprint matching using the distribution of the pairwise distances between minutiae. In AVBPA, pages 693-701, 2005.

[13] S. Raudys and A. Jain. Small sample size effects in statistical pattern recognition: Recommendations for practitioners. 13(3):252-264, March 1991.

[14] A. Ross, J. Shah, and A. K. Jain. From template to image: Reconstructing fingerprints from minutiae points. IEEE Transactions on Pattern Analysis and Machine Intelligence, 29(4):544-560, 2007.

[15] P. Tuyls, A. Akkermans, T. Kevenaar, G. Schrijen, A. Bazen, and R. Veldhuis. Practical biometric authentication with template protection. In AVBPA, pages 436-446, 2005.

[16] A. Willis and L. Myers. A cost-effective fingerprint recognition system for use with low-quality prints and damaged fingertips. Pattern Recognition, 34(2):255-270, 2001.

[17] H. Xu and R. Veldhuis. Spectral minutiae representatoins of fingerprints enhanced by quality data. In IEEE Third International Conference on Biometrics: Theory, Applications and Systems (BTAS '09), September 2009.

[18] H. Xu, R. Veldhuis, A. Bazen, T. Kevenaar, T. Akkermans, and B. Gokberk. Fingerprint verification using spectral minutiae representations. Information Forensics and Security, IEEE Transactions on, 4(3):397-409, Sept. 2009.

[19] H. Xu and R. N. J. Veldhuis. Spectral representations of fingerprint minutiae subsets. In Image and Signal Processing, 2009. CISP '09. 2nd International Congress on, pages 1-5, Oct. 2009.

[20] H. Xu, R. N. J. Veldhuis, T. A. M. Kevenaar, A. H. M. Akkermans, and A. M. Bazen. Spectral Minutiae: A Fixed-length Representation of a Minutiae Set. In Proceedings of the IEEE Computer Society Conference on Computer Vision and Pattern Recognition - Workshop on Biometrics, Anchorage, USA, 2008. 